\title{
Optimization flow model for handling car flows at the marshalling yard
}

\author{
Alexander Alexandrov ${ }^{1, *}$, Alexander Galkin ${ }^{1}$, Elena Timukhina ${ }^{1}$, and Nikolai Tushin ${ }^{1}$ \\ ${ }^{1}$ Ural State University of Railway Transport, 620034 Yekaterinburg, Russia
}

\begin{abstract}
Mathematical modeling is widely used in studies of operational planning in railway transport. Mainly heuristic algorithms and simulation modeling is used in short-term planning at marshalling yards. There is an opportunity to improve the quality of planning using strict optimization models. The objective of the study was to find a way to optimize the multiterminal transport flows of the complex structure by the example of operational planning for handling car flows at the marshalling yard. The process model was built on the basis of Mixed Integer Linear Program (MILP) method. The article provides the mathematical setting and describes results of calculations of the reference example. The LpSolve application package was used. The technological effect is estimated through checking the optimal solution on the simulation model.
\end{abstract}

\section{Introduction}

A linear programming device is traditionally used in solving problems of optimal transport flows management. However, the most developed formulations of the transport problem and the general problem of linear programming do not always allow taking important technological limitations into consideration. Some restrictions may require modeling of logical relationships with conditions of the "either-or" type. Such conditions can be taken into consideration by means of binary variables (taking value 0 or 1). In this case, the problem goes into the category of mixed integer linear programming (MILP) problems.

Publications with examples of the use of various MILP task settings to solve practical problems in management of transport flows have often appeared in recent years [1,2].

The paper [1] is devoted to compilation and updating of set schedules in the railway network in the southern region of Switzerland. The results showed that the proposed MILP allows finding the optimal solution quite quickly in many cases.

Work [2] is considering an alternative mode of delivery of goods using the urban railway infrastructure. Instead of using trucks to transport goods, the possibility of using the existing suburban railway network is being explored. The MILP model is proposed to minimize the waiting time for daily deliveries. Numerical experiments have shown that the model is able to provide solutions for cases of moderate dimension in a very short time.

See the survey of studies related to the application of various models to solve planning problems in railway systems in [3] and [4].

\footnotetext{
${ }^{*}$ Corresponding author: alalexan@gmail.com
} 
Among research works of Russian authors aimed at studying the applicability of MILP to solve problems in the railway industry, a detailed study [5] shall be referred to. It is devoted to building models and methods for solving problems arising in planning of freight railway transport. Depending on the restrictions on locomotives, load-carrying capacity of sets, various algorithms for solving the problems of forming sets and traffic schedules for freight sets are proposed. The main difference from the problems of this article is the scale, i.e., a more distant planning horizon, a fundamentally larger object of management and a substantially smaller number of details of the structure and the technology of handling the transport flow at marshalling yards.

The objects of management at marshalling yards are sets and groups of cars in them. The flow of sets at the stage of breaking-up is transformed into a multitude of car flows that follow individual tracks of the marshalling yard. The transport problem of linear programming cannot adequately display such complex structure. This problem is solved by using simulation modeling $[6,7]$. In this case, it is possible to take into consideration various technological limitations, but strict optimization is not available. In this case, the authors see a good opportunity for applying MILP.

The objective of the study is finding a way to optimize the multi-terminal transport flows of the complex structure by the example of operational planning for handling car flows at the marshalling yard. It is necessary to minimize the time of cars staying at the station and ensure departure of sets according to the specified schedule. The authors used the MILP to build the model. To validate the model, the data from a real marshalling yard was used, and the solution was tested on a simulation model that functions in the automated control system (ACS) of the marshalling yard. The problem formulated in the article has not been solved earlier with the use of the apparatus of mixed integer linear programming.

\section{Research methods}

It is proposed to use the method of mixed integer linear programming for building the operational planning model for handling car flows at the marshalling yard. The description of the model is built in accordance with the methodology described in [8]. At the first stage, we will define the boundaries of the system simulated and the structure of the model. A flowchart will be used for this purpose. We will select the stages of handling the sets and the car flows at the phases of preparation for breaking-up, breaking-up and accumulation as blocks of the process.

The block "Waiting for preparation for breaking-up" is intended for displaying unproductive idle time due to employment of the survey team. The number of these blocks is limited by the number of receiving tracks. The block "Preparation for breaking-up" simulates the main operation at the station receiving yard. Availability of the block "Waiting for breaking-up" block is due to the mismatch between the rhythms of the subsystem of preparation for breaking-up and the subsystem of breaking-up. The "Breaking-up" block displays the handling capacity of the sorting hump through the technological interval of the hump. The "Accumulation" block reproduces the process with the same name. The number of these blocks corresponds to the number of set destinations formed by the station.

The work of the first pair of blocks in the model is in many ways fundamentally identical to the second pair of blocks. To shorten the descriptive part and reduce the number of components of the balance equations and the objective function, we will confine ourselves to describing the functioning of three types of the last blocks. The type of the abbreviated block diagram of the process of handling car flows at the marshalling yard is shown in Figure 1. 
This scheme is a static mapping of the model structure. Planning also involves considering the process, taking into consideration how it proceeds over time. This is implemented by increasing the dimensionality of the model in accordance with the number of clock cycles of the calculation period. The duration of the hump technological interval is used as the clock cycle. This parameter is defined as the average time spent on breaking-up of one train. The calculation period is connected with the current planning horizon of 4 hours. For example, if the hump technological interval is 15 minutes, then the calculation period is 16 cycles. The description of the principles and examples of transformation of the static network into a dynamic one can be found in $[9,10]$. We will add additional index $t, T$ $=[1, T]$ corresponding to the cycle of the calculation period into the notation of flows in the scheme (Fig. 1).

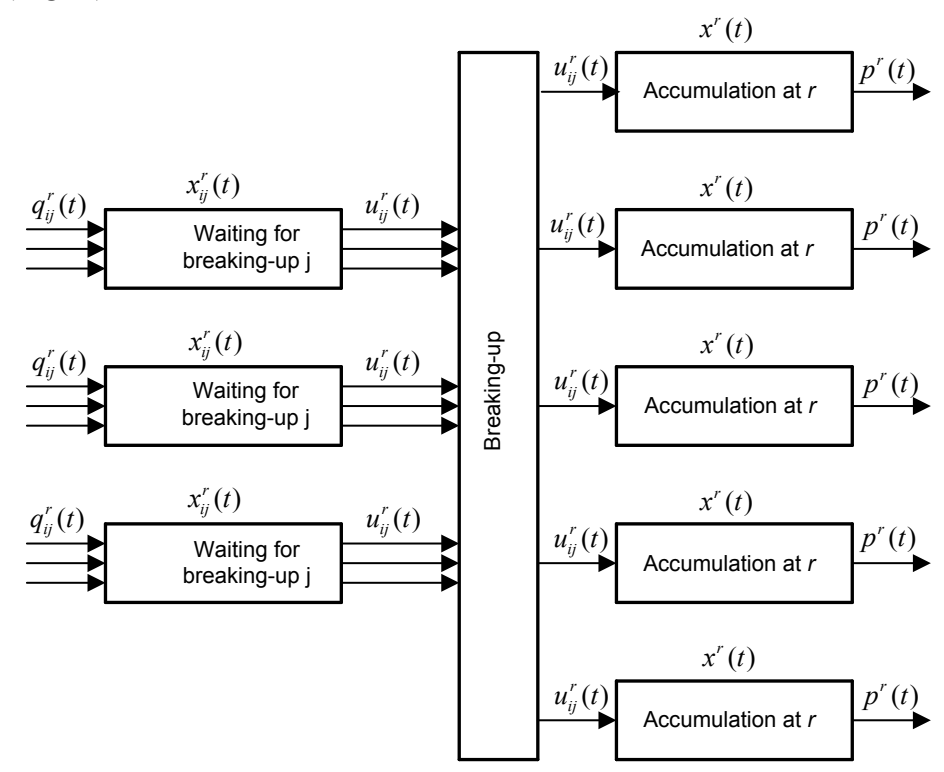

Fig. 1. Block diagram of the car flow handling at the marshalling yard.

Let us list the variables that we will use when writing the objective function and constraints:

$q_{i j}^{r}(t)$ - model input. The number of cars of destination $r$ in the $i$-th set, which comes from the "Preparation for breaking-up" block. These values are the source data.

$x_{i j}^{r}(t+1)$ is the required variable. The number of cars of destination $r$ in the $i$-th set, which is included into the $j$-thblock "Waiting for breaking-up" within cycle $t$;

$u_{i j}{ }^{r}(t+1)$ is the required variable. The number of cars of destination $r$, which, after breaking-up of the $i$-th set of the $j$-th block "Waiting for breaking-up" goes to block "Accumulation at $r$ " by the cycle commencement $(t+1)$;

$x^{r}(t+1)$ is the required variable. The number of cars of destination $r$, which stayed at block "Accumulation at $r$ " during cycle $t$;

$p^{r}(t+1)$ is the model output. The number of cars of destination $r$, which leaves the system at the end of system $t$. These values are the sourse data and they are meaningfully associated with the departure of sets according to the schedule.

The flow from the model input or from the "Waiting for breaking-up" block can get into the "Breaking-up" block or be delayed in the "Waiting for breaking-up" block. This 
circumstance shifts the problem to the category of mixed integer linear programming problems. The use of binary Boolean variables is required.

Let $y_{i j}(t)=\{0,1\}$ determine if there is delay of the $i$-th set in the $j$-th block "Waiting for breaking-up". In this turn, $z_{i j}(t)=\{0,1\}$ determines the result of taking the resolution of sending the $i$-th set from the $j$-the block "Waiting for breaking-up" to the "Breaking-up" block. In order to select only one of the two possible alternatives, the constraint of the following type should be used:

$$
y_{i j}(t)+z_{i j}(t)=1
$$

Next, let us write down constraints of the balance equations type.

$$
\begin{gathered}
\square r \mid y_{i j}(t-1) x_{i j}^{r}(t)+q_{i j}^{r}(t)-z_{i j}(t) u_{i j}^{r}(t+1)-y_{i j}(t) x_{i j}^{r}(t+1)=0 \\
\square i, j, t \mid \sum_{r=1}^{R} q_{i j}^{r}(t)=Q_{i j},
\end{gathered}
$$

where $Q_{i j}$ is the general value of the $i$-th set on the input into the $j$-th block "Waiting for breaking-up".

The following two restrictions will ensure breaking-up with entire trains. If there are no such restrictions, car groups will arrive in blocks "Accumulation at $r$ " in random order from different tracks within one clock cycle $t$, which contradicts the actual technology of breaking-up in entire trains.

$$
\begin{aligned}
& \square i, j, t \mid \sum_{r=1}^{R} u_{i j}^{r}(t+1)=z_{i j}(t) Q_{i j} \\
& \square i, j, t \mid \sum_{r=1}^{R} x_{i j}^{r}(t+1)=y_{i j}(t) Q_{i j}
\end{aligned}
$$

The following limitation will ensure the balance of cars in blocks "Accumulation at $r$ ":

$$
\square r \mid x^{r}(t)-p^{r}(t+1)+u_{i j}^{r}(t+1)-x^{r}(t+1)=0
$$

Restrictions on the throughput of arcs that represent the structure of a mathematical formulation and the non-negativity of variables are standard for this kind of problem, and they will not be presented here.

The objective function is the total cost of handling the car flows in the simulated system. It must be minimized.

$$
\Omega=\sum_{i=1}^{I} \sum_{j=1}^{J} \sum_{r=1}^{R} \sum_{t=1}^{T} c_{i j}{ }^{r} x_{i j}{ }^{r}(t)+\sum_{i=1}^{I} \sum_{j=1}^{J} \sum_{r=1}^{R} \sum_{t=1}^{T} l_{i j}{ }^{r} u_{i j}{ }^{r}(t+1)+\sum_{r=1}^{R} \sum_{t=1}^{T} m^{r} x^{r}(t+1),
$$

where $c_{i j}{ }^{r}, l_{i j}{ }^{r}, m^{r}$ are unit costs for one car of a certain purpose in one clock cycle at the respective handling stage. It is known that the operating costs for railway transport are proportional to the time required. In the simplest form, the listed unit costs can be taken as equal to one. Substantially, this will mean that for all sets and destinations at all stages of handling, priority is not taken into consideration. By changing these values, for example, by increasing the cost of idle time in the "Waiting for breaking-up" blocks, conditions for speedy movement of cars to the marshalling yard will be created. If this is done only for 
individual $r$ sets, they will complete the accumulation at the marshalling yard earlier than others. The order of breaking-up will be changed. Thus, the model is able to reproduce the real methods of controlling car flows handling. Methodological principles of solving mixed integer linear programming problems can be found in many sources $[8,11-13]$.

\section{Study results}

The numerical test case was solved using the LP SOLVE software on a PC with an Intel Core $17-4790 \mathrm{~K}$ processor. The size and structure of the model display the structure and technology of the Kamensk-Uralsky regional marshalling yard and has the following parameters: the number of variables is 841 , the number of limitations is 585 . The average calculation time was 386 seconds in a series of 30 calculations simulating the work of the yard for 5 days. Validation of the solution of the optimization problem was performed on the simulation model used in the automated control system by the marshalling yard [6]. Convergence of results of both types of models suggests that the optimization model behaves like a real system. The simulation model was also used to conduct experiments for simulation of planning based on the principles that guide the dispatcher in their work. The results of this simulation were used for comparison with the first series of calculations.

The technological effect, which is the basis of economic efficiency, can be assessed by improvement of such important indicator of the marshalling yard operation as the accumulation parameter. Decrease in the value of the accumulation parameter from 9.6 to 8.9 indicates that the decisions to choose the order of breaking-up of the formulations that the model proposes are more efficient.

\section{Conclusion}

An important feature of the proposed MILP optimization model is that it managed to correctly display the complex structure of the transport flow and its transformation while handling at the marshalling yard. With that, the time of cars staying at the marshalling yard is minimized and scheduled departure of sets is ensured.

It is possible to use this model in the automated control system of the marshalling yard, which will increase the level of certainty of a complex transport system, improve the natural and economic performance.

When moving to the study of larger marshalling yards with more than sixteen tracks, the volume of input information and the dimension of the problem itself will be increased substantially. It will be necessary to develop algorithms for automated generation of the initial data package for the problem. It will also be necessary to pay attention to optimizing the structure of the model itself and searching for effective solution algorithms for largedimensional problems. These issues determine the direction of future research.

\section{References}

1. F.F. Khoshniyat, J.T. Krasemann, $17^{\text {th }}$ Workshop on Algorithmic Approaches for Transportation Modelling, Optimization, and Systems (ATMOS 2017), 10, 1-17 (2017)

2. W. Behiri, O. Ozturk, S. Belmokhtar-Berraf, $6^{\text {th }}$ International Conference on Information Systems, Logistics and Supply Chain, 2, 1-8 (Bordeaux, France, June 1 4, 2016).

3. V. Cacchiani, D. Huisman, M. Kidd, L. Kroon, P. Toth, L. Veelenturf, J. Wagenaar, Transportation Research. Part B: Methodological, 63, 15-37 (2014). 
4. R.M. Lusby, J. Larsen, M. Ehrgott, D. Ryan, OR spectrum, 33(4), 843-883 (2011).

5. A.A. Lazarev, E.G. Musatova, E.R. Gafarov, A.G. Kvaracheliya, Teorija raspisanij. Zadachi zheleznodorozhnogo planirovanija [Scheduling theory. Tasks of railway planning] (Moscow, 2012). (in Russian)

6. A.E. Alexandrov, P.A. Kozlov, Zheleznodorozhnyj transport [Railway transport], 9, 65-67 (2003). (in Russian)

7. A.E. Alexandrov, S.V. Surin, Transport of the Urals, 4, 66-70 (2016). (in Russian)

8. B.A. Murtagh, Advanced Linear Programming: Computation and Practice (McGrawHill Intarnetional Book Company, 1981).

9. D.R. Ford, D.R. Fulkerson, Flows in Networks (Princeton University Press., New Jersey, 1962).

10. P.A. Kozlov, S.P. Milovidov, Izvestija AN SSSR. Tehnicheskaja kibernetika [Engineering Cybernetics], 5, (1983). (in Russian)

11. A.H. Land, A.G. Doig, Econometrica, 28, 497-520 (1960).

12. R.S. Garfinkel, G.L. Nemhauser, Integer Programming (Willey, New York, 1972).

13. V.L. Beresnev, E.Kh. Gimadi, V.T. Dementyev, Ekstremal'nye zadachi standartizacii [Extreme problems of standardization] (Nauka, Novosibirsk, 1978). (in Russian) 